\title{
Quantum Effects in Water: Proton Kinetic Energy Maxima in Stable and Supercooled Liquid
}

\author{
Antonino Pietropaolo \\ Universitá degli Studi di Milano Bicocca, CNISM, clo Dipartimento di \\ Fisica "G. Occhialini", Piazza della Scienza 3, 20126 Milano, Italy and \\ Universitá degli Studi di Roma Tor Vergata, Dipartimento di Fisica and Centro NAST, Via Ricerca Scienti ca 1, 00133 Rome, Italy \\ Roberto Senesi ${ }^{\dagger}$ and Carla Andreani \\ Universitá degli Studi di Roma Tor Vergata, Dipartimento di Fisica and Centro NAST, Via Ricerca Scienti ca 1, 00133 Rome, Italy
}

Jerry Mayers

ISIS Neutron Source, Science Technology Facility Council, Chilton, Oxfordshire, OX11 OQX, UK

(Received on 5 March, 2009)

\begin{abstract}
A strong temperature dependence of proton mean kinetic energy was observed for liquid water around the density maximum and for moderately supercooled water. Line shape analysis of proton momentum distribution, determined from deep inelastic neutron scattering measurements, shows that there are two proton kinetic energy maxima, one at the same temperature of the macroscopic density maximum at $277 \mathrm{~K}$, and another one in the supercooled phase located around $270 \mathrm{~K}$. The maximum at $277 \mathrm{~K}$ is a microscopic quantum counterpart of the macroscopic density maximum, where energetic balance giving rise to the local water structure is manifest in the temperature dependence of kinetic energy. The maximum in the supercooled phase, with higher kinetic energy with respect to stable phases, is associated to changes in the proton potential as the structure evolves with a large number of $\mathrm{H}$-bond units providing both stronger effective proton localization, as well as proton quantum delocalization.
\end{abstract}

Keywords: momentum distribution; hydrogen bond; supercooled water; density maximum

The study of the microscopic dynamics of liquid water is a challenge of great interest due to the fundamental relevance of this system in biological and physical sciences [1]. This simple liquid still represents a challenging puzzle [2-4]. A crucial piece of information is the understanding of the local environment of the protons which plays a fundamental role in hydrogen bonding. Recent studies on the microscopic structure [4] and dynamics of water [5-9] have shown that quantum effects associated with protons have a significant impact on the behavior of hydrogen bond networks. Quantum effects, such as those associated with the breaking and distortion of hydrogen bonds [5, 7, 9, 10], are best revealed by measurements of proton momentum distributions, $n(p)$, and mean kinetic energy, $\left\langle E_{K}\right\rangle[11]$.

The latter quantity provides unique information and details on the effective potential that the proton sees as the hydrogen bond network changes, such as proton tunneling and delocalization, thus complementing the well studied spatial distributions of the proton $[12,13]$.

We report direct measurements of the proton $\left\langle E_{K}\right\rangle$ and $n(p)$ in water, in a wide temperature range from stable to metastable liquid. The $\left\langle E_{K}\right\rangle$ shows two maxima: at 277 $K$ and in the supercooled phase in the range $269 \mathrm{~K}-271 \mathrm{~K}$, with an excess of $\left\langle E_{K}>\right.$ of about $21 \mathrm{meV}$ and $85 \mathrm{meV}$ higher than the value at $300 \mathrm{~K}$, respectively. The first maximum, at the same temperature of the macroscopic density maximum in liquid water [14], is a fingerprint of the cohesive nature of the hydrogen-bonded network. The second maximum, characterized by a much larger excess of $\left\langle E_{K}\right\rangle$, is a remarkable feature which is interpreted as a clear signature of nuclear

Electronic address: antonino.pietropaolo@mib.infn.it

${ }^{\dagger}$ Electronic address: roberto. senesi@roma2.infn.it quantum effects rising up in the supercooled phase [15].

The momentum distribution is a direct re ection of the shape of the proton potential, due to the quantum mechanical non commuting property of position and momentum. For example, differences in $n(p)$ between liquid water and ice re ect the breaking and distortion of hydrogen bonds that occurs upon melting [6,9].

From the experimental point of view, pulsed neutron sources have allowed the remarkable advance of Deep Inelastic Neutron Scattering (DINS) technique. At present DINS is the only technique which determines the proton momentum distribution, $n(p)$, and mean kinetic energy, $\left\langle E_{K}\right\rangle$. It is based on neutron scattering measurements at high energy $(1 \mathrm{eV}<\hbar<200 \mathrm{eV})$ and wave vector, $\left(20 \AA^{-1}<q\right.$ $\left.<300 \AA^{-1}\right)$ transfers. DINS probes both the short-time dynamics and local environment of the light atoms [11]. The high energy and wave vector transfers achieved allow the scattering process to be described within the framework of the Impulse Approximations (IA) [16]. This assumes that neutron scattering is incoherent and occurring within time scales much shorter than the typical relaxation times of the collective excitations. In these conditions the struck particle recoils freely from the collision, with inter-particle interaction in the final state being negligible (i.e. the wave function of the particle in its final state assumed to be a plane wave). The scattering cross section is then expressed in terms of the single particle momentum distribution $n(p)$, whose variance is in turn related to the single particle mean kinetic energy, $\left.<E_{K}\right\rangle$. In the case of water protons for instance, $n(p)$ provides a richness of information about the potential surface that the proton experiences, including the effects of hydrogen bonding. DINS complements microscopic structural studies and allows a quantitative comparison with quantum Monte Carlo simulations $[8,9]$. These possibilities and a variety of successful experiments on light atoms and molecules make 
DINS technique a unique and well established tool to investigate the hydrogen bonding [17] and intermolecular structure of water under various conditions [11].

The DINS experiment has been carried out on the VESUVIO spectrometer [18], at the ISIS spallation neutron source (Rutherford Appleton Laboratory, UK). The bulk water samples (bi-distilled and de-ionized) were contained in a disk shaped aluminum can $(5 \mathrm{~cm}$ diameter, $1 \mathrm{~mm}$ sample thickness) with inner $\mathrm{Te}$ on coating to prevent crystallization. The occurrence of Bragg's peaks in the thermal neutron energy range was constantly monitored, during data acquisition, to check for freezing. Two platinum temperature sensors, placed in the aluminum can walls close to the sample, monitored heat release due to ice formation when the sample was below $273 \mathrm{~K}$. No Bragg's peaks signals nor latent heat release signals due to solidification were observed for the two measurements at $T=272.15 \mathrm{~K}$ and $T=272.95 \mathrm{~K}$. Experimental data have been recorded by 64 fixed-angle detectors in the Resonance Detector configuration [19, 20] employing the Foil Cycling technique (FCT) [21]. Data have been corrected for multiple scattering, aluminum and oxygen signals. At high energy, $\hbar$, and high momentum, $\hbar q$, transfers neutron scattering is well described within the framework of the Impulse Approximation (IA) [11]. Within the IA framework the dynamical structure factor is related to the $n(p)$ through the relation:

$$
S_{I A}(q)=\int n(p) \quad\left(-\frac{\hbar q^{2}}{2 m}-\frac{p q}{m}\right) d p
$$

where $\hbar$ is the energy transfer, $\frac{\hbar^{2} q^{2}}{2 m}$ is the recoil energy of the struck atom of mass $m$ and $q$ is the wave vector transfer. The scattering law is then expressed in terms of a Neutron Compton Profile (NCP) [11]: $F(y)=\frac{\hbar q}{m} S_{I A}(q \quad)$, where $y=$ $\frac{m}{\hbar q}\left(-\frac{\hbar q^{2}}{2 m}\right)$ is the West scaling variable16. $F(y)$ is then represented as a series expansion in Hermite polynomials, where the small corrections due to deviations from the IA are taken into account. The NCP line shape was then convolved with the instrumental resolution function and the coefficients in the series expansion determined by a least squares fitting procedure. The momentum distribution is then expressed by the expansion:

$$
n(p)=\frac{e^{-\frac{p^{2}}{2^{2}}}}{\left(\overline{2 \pi^{2}}\right)^{3}}{ }_{n} C_{n}(-1)^{n} L_{n}^{12}\left(\frac{p^{2}}{2^{2}}\right)
$$

$L_{n}^{12}(x)$ being the generalized Laguerre polynomials, is the standard deviation of the NCP, and $C_{n}$ are the coefficients of the series expansion associated to non-gaussian components in $n(p)$. Full details on the procedure to extract the momentum distribution $n(p)$ can be found in ref. [11].

Results of $\left\langle E_{K}>\right.$ from the present study and from previous measurements are plotted in Figure 1 and reported in Table I. The Figure reports $\left\langle E_{K}\right\rangle$ values in the solid [6], in the metastable phase - supercooled phase from $T=269.00 \mathrm{~K}$ [15] to $T=272.95 \mathrm{~K}$ (present data) - in the region of the density maximum from present study - at $T=274.15 \mathrm{~K}$, $T=277.15 \mathrm{~K}, T=281.15 \mathrm{~K}, T=285.15 \mathrm{~K}$, at room temperature $[6,7,11]$ up the supercritical point $[5,7]$. The $\left\langle E_{K}>\right.$ exhibits two local maxima, one in the stable liquid phase at
TABLE 1: Values of water proton mean kinetic energy, $\left\langle E_{K}\right\rangle$, in the stable liquid, solid, supercritical and supercooled phases from present and previous measurements. Uncertainties in the $\left\langle E_{K}\right\rangle$

values are derived from the least squares fitting procedure parameter errors $[7,10,11]$. Temperature uncertainties is $0.01 \mathrm{~K}$ in all measurements. Values of water density are also reported for ice [22], supercooled phase [23, 24], stable liquid and supercritical uid [25].

\begin{tabular}{|c|c|c|c|c|}
\hline & $\mathrm{T}(\mathrm{K})$ & $<\mathrm{E}_{\mathrm{K}}>(\mathrm{kJ} / \mathrm{mole})$ & $<\mathrm{E}_{\mathrm{K}}>(\mathrm{meV})$ & $\rho\left(\mathrm{kg} / \mathrm{m}^{3}\right)$ \\
\hline Ice $^{6}$ & 269.00 & $12.5 \pm 0.3$ & $130 \pm 3$ & 916.70 \\
\hline Supercooled $^{15}$ & 269.00 & $19.2 \pm 0.2$ & $199 \pm 2$ & 999.41 \\
\hline Supercooled $^{15}$ & 271.00 & $22.0 \pm 0.3$ & $228 \pm 2$ & 999.67 \\
\hline Supercooled $^{*}$ & 272.15 & $16.1 \pm 0.3$ & $167 \pm 2$ & 999.76 \\
& & & & \\
\hline Supercooled $^{*}$ & 272.95 & $14.5 \pm 0.3$ & $150 \pm 2$ & 999.84 \\
\hline Stable $^{*}$ & 274.15 & $15.0 \pm 0.1$ & $156 \pm 1$ & 999.90 \\
\hline Stable $^{*}$ & 277.15 & $15.8 \pm 0.1$ & $164 \pm 1$ & 999.97 \\
\hline Stable $^{*}$ & 281.15 & $15.6 \pm 0.1$ & $162 \pm 1$ & 999.85 \\
\hline Stable $^{*}$ & 285.15 & $15.4 \pm 0.1$ & $160 \pm 1$ & 999.50 \\
\hline Stable $^{19}$ & 293.00 & $14.2 \pm 0.8$ & $147 \pm 8$ & 998.19 \\
\hline Stable $^{6}$ & 296.15 & $14.1 \pm 0.3$ & $146 \pm 3$ & 997.50 \\
\hline Stable $^{7}$ & 300.00 & $13.8 \pm 0.3$ & $143 \pm 3$ & 999.51 \\
\hline Stable $^{7}$ & 423.00 & $14.9 \pm 0.3$ & $155 \pm 3$ & 922.46 \\
\hline Stable $^{7}$ & 523.00 & $16.3 \pm 0.5$ & $169 \pm 5$ & 802.03 \\
\hline Stable $^{7}$ & 573.00 & $16.6 \pm 0.5$ & $172 \pm 5$ & 719.88 \\
\hline Supercritical $^{7}$ & 673.00 & $17.2 \pm 0.4$ & $178 \pm 4$ & 701.65 \\
\hline & & & & \\
\hline & & & & \\
\hline & & & & \\
\hline & & & & \\
\hline & & & & \\
\hline & & & & \\
\hline & & & & \\
\hline
\end{tabular}

* present measurements

about $\mathrm{T}=277.15 \mathrm{~K}$ and the other at about $271 \mathrm{~K}$. The maxima occur at the temperature of density maximum of liquid water and in the supercooled phase, with $\left\langle E_{K}\right\rangle$ in excess of about $21 \mathrm{meV}(2.2 \mathrm{~kJ} / \mathrm{mole})$ and $85 \mathrm{meV}(8.2 \mathrm{~kJ} / \mathrm{mole})$ to the room temperature value, respectively. The excess of proton $<E_{K}>$ is interpreted as an increasing confinement of protons as the density maximum is approached from freezing temperature to $277.15 \mathrm{~K}$. The proton $\left\langle E_{K}\right\rangle$ excess is the microscopic counterpart of the macroscopic one, brought about by the opposing effects of increasing temperature, causing both structural collapse that increases density and thermal expansion that lowers density. Moreover a remarkable excess of proton $\left\langle E_{K}\right\rangle$ is found in the metastable state, between 269 $K$ and $273 K$. While an increase in $\left\langle E_{K}\right\rangle$ in supercooled water with respect to ice is not unexpected, bearing in mind that this would take into account the kinetic energy contribution to the latent heat of solidification, the existence of a maximum is suggested by the present results. This is interpreted as the fingerprint of the quantum nature of the protons, in terms of the modification of the potential they experience as entering in the metastable phase, with a maximum of about $52 \%$ excess of the $\left\langle E_{K}\right\rangle$ (see Table I). The latter is very close to the H-bond breaking energy, i.e. about $7 \mathrm{~kJ} / \mathrm{mole}$. The excess with respect to the value at room temperature is even greater, about 59\%. Figure 2 reports $\left\langle E_{K}\right\rangle /$ versus $T$, where is the density, and highlights the excess of $\left\langle E_{K}\right\rangle$ in the metastable state with respect to stable phases. In the stable phase around the density maximum, $\left\langle E_{K}\right\rangle$ increases with density, while an even larger $\left\langle E_{K}\right\rangle$ is found in the metastable phase. In the latter, counter-intuitively, the dis- 
tance between the water molecules decreases [15] as the density decreases, the decrease in density being primarily due to the reduction in nearest neighbors. The temperature dependence of $\left\langle E_{K}\right\rangle$ in the stable phases can be well described taking into account translational, rotational, and vibrational contributions, and making use of the optical spectroscopic data available in the literature $[5,11]$. In this framework the $\left\langle E_{K}\right\rangle$ turns out to vary almost linearly with $T$, although with a gradient lower then $3 / 2 k_{B} T$ [17]. This dependence fits very nicely the set of $\left\langle E_{K}\right\rangle$ for water data, from the solid state through room temperature up to supercritical state $[5,7]$, with the exclusion of the region of density maximum and in the supercooled state. Indeed present data clearly indicates that linear behavior of $\left\langle E_{K}\right\rangle$ versus $T$ is lost in both the stable phase, around the density maximum, and in the metastable state (see Figure 1).

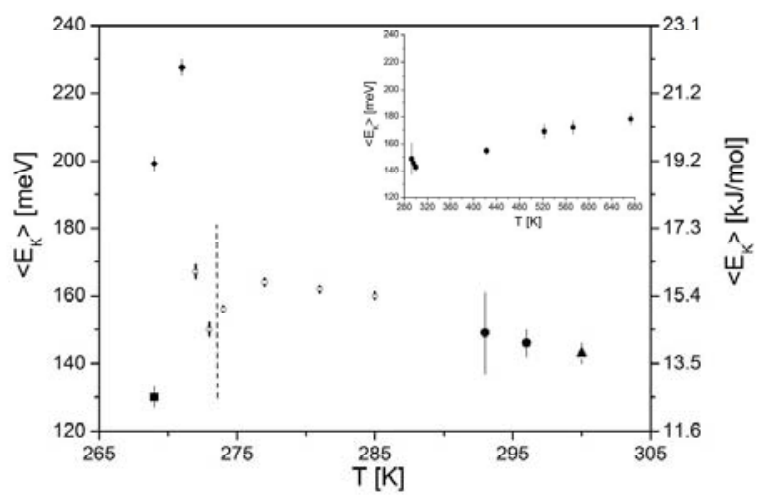

FIG. 1: Proton mean kinetic energy $\left\langle E_{K}\right\rangle$ in water as a function of temperature. From left to from left to right: Ice [6] at $269.00 \mathrm{~K}$ (squares); metastable supercooled water from ref. [15] (diamonds) at $T=269.00 \mathrm{~K}$ and $T=271.00 \mathrm{~K}$ and from present experiment (open circles) $T=272.15 \mathrm{~K}$; stable water from present experiment (open circles) at $T=272.95 \mathrm{~K}, T=274.15 \mathrm{~K}, T=277.15 \mathrm{~K}, T=$ $281.15 \mathrm{~K}, T=285.15 \mathrm{~K}$, and from refs. [19,6] (full circle) at $T=$ $293.00 \mathrm{~K}, T=296.15 \mathrm{~K}$ and from ref. [7] (triangle) at $T=300.00$ $\mathrm{K}$. Insert shows proton $\left\langle E_{K}\right\rangle$ for stable water at $T=423.00 \mathrm{~K}$, $T=523.00 \mathrm{~K}, T=573.00 \mathrm{~K}, T=673.00 \mathrm{~K}$ from ref. [7]. Dashed line marks the position of the triple point. The error bars are derived from the least squares fitting procedure parameter errors [7, 10, 11]

Similar remarkable features are found in the $n(p)$. A plot in Figure 3 of the radial momentum distributions $4 \pi \mathrm{p}^{2} n(p)$ most effectively highlights the differences between the $n(p)$ 's measured in the different states. The proton $\left\langle E_{K}>\right.$ and $n(p)$ experimental results demonstrate the central role played by hydrogen bonding in determining the dynamical properties of water. In the stable phase, the proton dynamics in the region of the density maximum is determined by the balance between the weakening of the covalent bond (corresponding to a more delocalized proton) and the increasingly organized and open four-coordinated network of hydrogen bonds. In the metastable phase the excess of $\left\langle E_{K}\right\rangle$ and the $n(p)$ line shapes provide quantitative information on the quantum nature of proton and on the changes occurring in the potential experienced by protons.

The $\left\langle E_{K}>\right.$ excess and the changes in line shapes of the $n(p)$ in the metastable phase are interpreted as follows. The



FIG. 2: The $<E_{K}>/ r$ ratio in water as a function of temperature for the thermodynamical points listed in Table I and plotted in Figure 1. Dashed line marks the temperature of the triple point. The error bars are derived from the least squares fitting procedure parameter errors $[7,10,11]$.

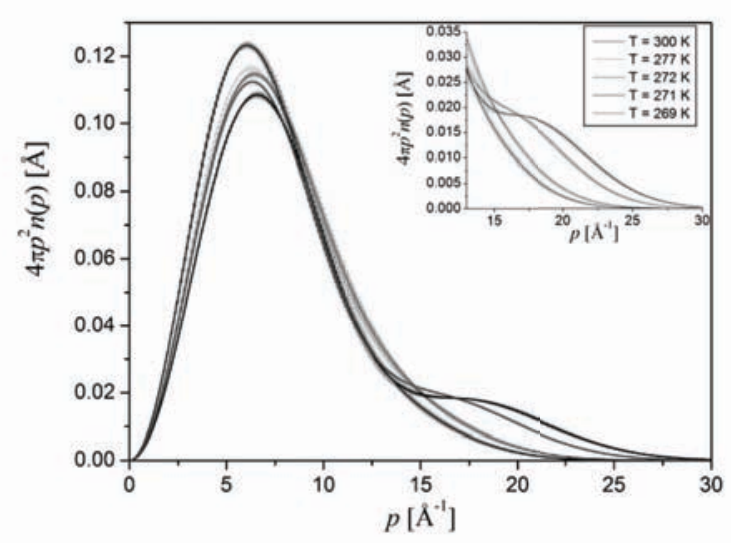

FIG. 3: Radial proton momentum distribution, $4 p^{2} n(p)$, for water as a function of $p$. Stable phase, at room temperature, $T=300.00$ $\mathrm{K}$ [4] (blue line) at the temperature of the density maximum, $T=$ $277.15 \mathrm{~K}$ (green line), in the metastable supercooled phase, at $T=$ 272.15 K [4] (red line), $T=271.00 \mathrm{~K}$ (black line) and $T=269.00$ $\mathrm{K}$ (purple line). The error bars are less than $1 \%$ and were derived from the least squares fitting procedure parameter errors [7, 10, 11]. The additional intensities in the wings of the data at $T=271.00 \mathrm{~K}$ (black) and $T=269.00 \mathrm{~K}$ (purple), must be due to the protons being more localised in the supercooled phase. The minimum in $4 \pi p^{2} n(p)$ at $\sim 14 \AA^{-1}$ suggests that the protons are confined in double well hydrogen bond potentials [15].

H-bond network is formed with a greater number of protons, and on average the protons experience an increased localisation. The hydrogen bond network in supercooled water responds to localisation by developing effective double well (or nearly so) potentials leading to coherent motion of the protons in the two wells, thus in a narrowing of $n(p)$ at low momenta and broadening at high momenta.

These results, in addition to revealing the local proton dynamic of the bond, provide a data base for $a b$ initio calculations of hydrogen bond potentials that is non-existent at the moment. 


\section{Acknowledgements:}

This work was supported within the CNR-CCLRC Agreement No. 01/9001 concerning collaboration in scientific re- search at the spallation neutron source ISIS. The financial support of the Consiglio Nazionale delle Ricerche in this research is hereby acknowledged. We also acknowledge useful discussions with F. Bruni, D. Flammini, M. A. Ricci.
[1] D. Eisenberg, W. Kauzmann. The Structure and Properties of Water (Oxford University Press, Oxford, 1969).

[2] O. Mishima, H.E. Stanley, Nature 396, 329-335 (1998).

[3] P.L.Geissler, C. Dellago, D. Chandler, J. Hutter, M. Parrinello, Science 291, 2121-2124 (2001).

[4] A.K. Soper, C.J. Benmore, Phys. Rev. Lett. 101, 065502 (2008).

[5] C. Andreani, D. Colognesi, E. Degiorgi, M. A. Ricci, J. Chem. Phys. 115, 11243-11248 (2001).

[6] G. F. Reiter, J. C. Li, J. Mayers, T. Abdul-Redah, P. Platzman, Braz. J. Phys. 34, 142-147 (2004).

[7] C. Pantalei, et al., Phys Rev. Lett. 100, 177801 (2008).

[8] J.A. Morrone, V. Srinivasan, D. Sebastiani, R. Car, J. Chem. Phys. 126, 234504-234512 (2007).

[9] J.A. Morrone, R. Car, Phys. Rev. Lett. 101, 017801 (2008).

[10] V. Garbuio, et al. , J. Chem. Phys. 127, 154501-154510 (2007).

[11] C. Andreani, D. Colognesi, J. Mayers, G. F. Reiter, R. Senesi, Adv. Phys. 54, 377-469 (2005).

[12] A.K. Soper, J. Phys. Condens. Matter 19, 335206-335224 (2007).

[13] A. Botti, et al., J. Chem. Phys. 117, 6196-6199 (2002).
[14] H. Tanaka, Phys. Rev. Lett. $805750-5753$ (1998).

[15] A. Pietropaolo, , et al. , Phys. Rev. Lett. 100, 127802 (2008).

[16] G.B. West, Phys. Rep., 18, 263-323 (1975).

[17] G.F. Reiter, J. Mayers, J. Noreland, Phys. Rev. B., 65, $104305-$ 104313 (2002)

[18] J. Mayers, et al., Physica B: Condensed Matter, 350, E659E662 (2004).

[19] A. Pietropaolo, et al. , JINST, 1 P04001 doi: 10.1088/17480221/1/04/P04001 (2006) .

[20] C. Andreani, et al. , App. Phys. Lett. 85, 5454-5456 (2004).

[21] E.M. Schooneveld, et al. , Rev. Sci. Inst. 77, 095103 (2006).

[22] R. C.Weast, Handbook of Chemistry and Physics (CRC Press, Inc, Cleveland, 1975).

[23] G.S. Kell, J. Chem. Eng. Data 20, 97-105 (1975).

[24] G.S. Kell, J. Chem. Eng. Data 12, 66-69 (1967).

[25] E.W Lemmon,. M.O. McLinden, D. G. Friend, Thermophysical Properties of Fluid Systems. In NIST Chemistry WebBook, NIST Standard Reference Database Number 69, Eds. Linstrom, P.J. \& Mallard, W.G., National Institute of Standards and Technology, Gaithersburg MD, 20899, http://webbook.nist.gov, (retrieved January 8, 2009). 